\title{
Psychometric Performance of Learning Burnout Scale for Under- graduates (LBSU) in Guangdong
}

\section{Yongmei Hou* Yiyang Wang}

Department of Psychology, School of Humanity and Administration, Guangdong Medical University, Dongguan, Guangdong, 523808, China

\begin{tabular}{|c|c|}
\hline ARTICLE INFO & ABSTRACT \\
\hline Article history & To analyze the psychometric performance of Learning Burnout Scale \\
\hline Received: 14 July 2021 & for Undergraduates (LBSU) in Guangdong province. LBSU was used to \\
\hline Reviced. 16 July 2021 & conduct the survey involving 1628 undergraduates who were selected \\
\hline Revised: 16 July 2021 & with stratified random sampling from 7 colleges in Guangdong province. \\
\hline Accepted: 20 July 2021 & Cronbach's $\alpha$ coefficient and split-half reliability were used to analyze the \\
\hline Published Online: 30 October 2021 & internal consistency of the questionnaire. Convergent validity, discriminant \\
\hline Keywords: & Ceiling and floor effect were used to analyze its sensitivity. Cronbach's $\alpha$ \\
\hline IBSU & coefficient of the total questionnaire was 0.89 and cronbach's $\alpha$ coefficient \\
\hline LDSU & of 3 dimensions were $0.73-0.78$, which met with the requirements of the \\
\hline Learning burnout & group comparison. Spearman - Brown split-half coefficient of the total \\
\hline Validity & questionnaire and 3 dimensions were $0.90,0.85,0.81,0.79$, respectively, \\
\hline Reliability & which also met with the requirements of the group comparison. Both the \\
\hline Pesnonsiveness & calibration success rate of convergent validity and discriminant validity \\
\hline Responsiveness & of each dimension were $100 \%$. Four components obtained from 20 items \\
\hline Undergraduates & $\begin{array}{l}\text { which cumulative variance contribution rate was } 51.924 \% \text {. The total score } \\
\text { and score of each dimension were all normal distribution, without any floor } \\
\text { or ceiling effect in dimensions. The psychometric performance of LBSU } \\
\text { for assessing undergraduates in Guangdong province is valid and reliable. }\end{array}$ \\
\hline
\end{tabular}

\section{Introduction}

Learning burnout refers to a kind of behavior that students feel frustrated, tired, depressed or tired because of long-term strong psychological pressure or lack of learning motivation or interest in learning. Learning burnout is a common learning problem of college students, with the detection rate of $9.9-40.3 \%$ beyond seas ${ }^{[1,2]}, 24.8-51.3 \%$ at home ${ }^{[3-5]}$, and increasing year by year ${ }^{[6]}$. Learning burnout reduces learning enthusiasm ${ }^{[7]}$, leads to physical and mental fatigue ${ }^{[8]}$ and psychological syndrome ${ }^{[9,10]}$, hinders academic ${ }^{[11]}$ and career development ${ }^{[12]}$.

As learning burnout is of great significance to college students' physical and mental health, it is increasingly concerned by social public.

For the study of learning burnout, foreign countries carried out earlier, and developed many assessment tools, which are widely used for students of all ages (children, adolescents and adults, etc) ${ }^{[13,14]}$. However, Western measurement tools do not cover the content of Asian learning burnout and the unique way of Asian behavior ${ }^{[15]}$. Therefore, it is necessary to develop a localized measurement tool for Asian learning burnout.

Learning Burnout Scale for Undergraduates (LBSU) ${ }^{[15]}$

*Corresponding Author:

Yongmei Hou,

Department of Psychology, School of Humanity and Administration, Guangdong Medical University, Dongguan, Guangdong, 523808, China;

Email:2184456621@qq.com 
is a local scale compiled by Lian Rong (2005). It has simple items, clear structure, and its content is suitable for the life and learning conditions of Asian college students. Since its preparation, it has been more and more widely used. But what is the psychometric performance of LBSU applied to college students? At present, there is a lack of relevant evidence.

Based on the above analysis, this study intends to explore the psychometric performance of LBSU applied to college students in Guangdong Province by using a large sample and multi center survey model.

\section{Objects and Methods}

\subsection{Objects}

\subsubsection{Sample Size Estimation}

The minimum sample size is calculated by $\mathrm{G} *$ power 3 ${ }^{[16]}$. As the detection rate of learning burnout among domestic college students is $24.8-51.3 \%^{[3-5]}$, the test effective value is medium level ${ }^{[3-5]}$, that is, the $\mathrm{d}$ value is $0.50-0.80^{[17]}$. In this study, we set the effect value $d=0.70$, the statistical test power of $1-\beta=0.80$, the type I error probability $\alpha=0.05$, and the minimum sample size is calculated as 634 . The minimum sample size is determined as 761 due to a $20 \%$ of possible follow-up loss rate.

\subsubsection{Sampling}

By stratified random sampling, undergraduates from freshmen to fifth year from 7 universities including Sun Yat-sen University, Guangdong University of Finance and Economics, Guangzhou College of South China University of Technology, Guangdong Medical University, Guangdong Ocean University, Guangzhou Institute of Physical Education, as well as Guangzhou Academy of Fine Arts were selected as the research objects. 1800 questionnaires were distributed and 1628 valid questionnaires were collected, with an effective rate of $90.4 \%$. The average age was $(20.8 \pm 3.9)$ years. There are 900 boys and 728 girls; 683 urban students and 939 rural students; 422 freshmen, 389 sophomores, 407 juniors, 317 seniors and 93 fifth year students; 663 engineering students, 357 science students, 391 liberal arts students, 135 art students and 82 sports students.

\subsection{Tools}

\subsubsection{Learning Burnout Scale for Undergraduates (LBSU) ${ }^{[15]}$}

It is complied by Lian Rong and Yang Lixian according to Maslash Job Burnout scale, which is belonged to selfevaluation scale and used to evaluate the learning burnout of college students. There are 20 items, divided into three dimensions: low mood (LD), improper behavior (IB) and low sense of achievement (LSA). The 5-point scoring method is used to score from 1 to 5 points corresponding to "completely inconsistent" to "completely consistent". The higher the score, the more obvious the tendency in the item, dimension or learning burnout.

\subsubsection{Self-compiled Personal Information Questionnaire}

It includes 4 items, namely, gender, grade, origin, college.

\subsection{Collection and Arrangement of Data}

Before the investigation, the researchers who participated in the survey were trained uniformly, and the investigation process and evaluation standard were unified. The consistency test (kappa $=0.81-0.90)$ meets the test requirements.

The questionnaires with scores of more than $50 \%$ of the items missing were eliminated. The missing values of the valid questionnaires were estimated and filled with the average. Two researchers independently input the same data using Epidata3.0 software and conduct a unified logic check to ensure the accuracy of the data.

\subsection{Statistical Methods}

Data were exported from epidata3.0 to SPSS 20.0 for statistical analysis. First, the original score of the total scale and each dimension are calculated. The second step is to get the average score and standard deviation of the total scale and each dimension. In the third step, the floor and ceiling effect were evaluated, and then, cronbach's $\alpha$ coefficient and Split half reliability were calculated. Finally, convergent validity, discriminant validity and principal component factor analysis was condu cted.

Table 1. Scoring method of LBSU

\begin{tabular}{cccc}
\hline Dimension & item number & item distribution & $\begin{array}{c}\text { range of } \\
\text { original score }\end{array}$ \\
\hline $\begin{array}{c}\text { Low mood (LM) } \\
\text { Improper behavior } \\
\text { (IB) }\end{array}$ & 8 & $2,4,5,7,9,12,17,20$ & $1-40$ \\
$\begin{array}{c}\text { Low sense of } \\
\text { achievement(LSA) }\end{array}$ & 6 & $3,6,11,13,15,18$ & $1-30$ \\
\hline
\end{tabular}

\section{Results}

\subsection{The Distribution of LBSCU Scores}

The ceiling / floor effect is one of the psychological test 
effects, which refers to the phenomenon that when a test is too simple / complex, the scores of most objects are close to or reach the upper / lower limit of scores, which makes the evaluation and prediction performance of the test decrease ${ }^{[17]}$. Table 2 shows that the total score of LBSU and the scores of 3 dimensions all tend to be normal distribution, without any floor effect or ceiling effect.

\subsection{Reliability Analysis of LBSU}

\subsubsection{Split Half Reliability}

The 20 items of LBSU are divided into two parts with 10 items each, and the correlation coefficients of these two parts is $0.77(P<0.01)$. According to Sperman Brown formula, the split half reliability of total scale is 0.90 . The correlation coefficients of the two halves of 3 dimensions are $0.77,0.75$ and 0.71 , and the split half reliability are $0.85,0.81$ and 0.79 , respectively.

\subsubsection{Internal Consistency Reliability}

Generally speaking, when Cronbach's $\alpha$ coefficient is greater than 0.7 , the internal consistency reliability is better. It can be seen from table 3 that the Cronbach's $\alpha$ coefficient of the total scale is 0.89 , and those of the 3 dimensions are $0.78,0.73,0.75$, respectively. There is a low to moderate correlation between each dimension, and a moderate to high correlation between each dimension and the total score of the scale. $(P<0.01)$.

\subsection{Validity Analysis of LBSU}

\subsubsection{Content Validity}

The correlation coefficient $(R)$ between each item and its dimension is used to represent the convergent validity. Generally, when $R \geq 0.4$, it can be considered that the convergent validity is better. Discriminant validity is expressed by the correlation coefficient between the item and other dimensions. If these correlation coefficients are lower than the correlation coefficient between the item and its dimension, the discriminant validity is better. The results show that the correlation coefficient between each item and its dimension was $>0.4$, which was higher than those correlation coefficients between the same item with other dimensions. The calibration success rates of convergent validity and discriminant validity were all $100 \%$. See Table 4.

Table 2. Descriptive Analysis of LBSU $(n=1628)$

\begin{tabular}{ccccccccc}
\hline Dimension Celling[n(\%)] & $\mathrm{X} \pm \mathrm{s}$ & Min & Max & P25 & P50 & P75 & Floor[n(\%)] \\
\hline LM & $21.72 \pm 5.35$ & 8.0 & 37.0 & 18.0 & 22.0 & 25.0 & $7(0.4)$ & $1(0.05)$ \\
IB & $17.97 \pm 3.93$ & 6.0 & 30.0 & 15.0 & 18.021 .0 & $2(0.1)$ & $1(0.05)$ \\
LSA & $17.04 \pm 3.77$ & 6.0 & 28.0 & 15.0 & 17.0 & 19.0 & $1(0.05)$ & $0(0)$ \\
Total score of LBSCU & $56.73 \pm 9.84$ & 23.0 & 93.0 & 51.0 & 57.0 & 63.0 & $0(0)$ \\
\hline
\end{tabular}

Table 3. Cronbach's a Coefficients and Pairwise Correlation Coefficients $(n=1628)$

\begin{tabular}{lcccc}
\hline \multicolumn{1}{c}{ Dimension } & Cronbach's $\alpha$ & 1 & 2 & 3 \\
\hline 1.LM & .78 & & & \\
2. IB & .73 & $.54^{* *}$ & & \\
3.LSA & .75 & $.17^{* *}$ & $.30^{* *}$ & $.81^{* *}$ \\
4 total score of LBSU & .89 & $.83^{* *}$ & $.59^{* *}$ \\
\hline
\end{tabular}

${ }^{* *} P<0.01$

Table 4. convergent validity and discriminant validity of LBSCU $(n=1628)$

\begin{tabular}{|c|c|c|c|c|c|c|}
\hline \multirow{2}{*}{ Dimension item number } & \multicolumn{3}{|c|}{ Discriminant vality } & \multicolumn{3}{|c|}{ Discriminant vality } \\
\hline & range of $R$ & success & success rate $(\%)$ & range of $R \mathrm{x}$ & success & success rate $(\%)$ \\
\hline $\begin{array}{c}\text { LD } \\
8\end{array}$ & $.445 \sim .682$ & $8 / 8$ & 100 & $.029 \sim .469$ & $16 / 16$ & 100 \\
\hline $\begin{array}{c}\text { IB } \\
6\end{array}$ & $.416 \sim .684$ & $6 / 6$ & 100 & $.010 \sim .498$ & $12 / 12$ & 100 \\
\hline $\begin{array}{c}\text { LSA } \\
6\end{array}$ & $.559 \sim .654$ & $6 / 6$ & 100 & $.012 \sim .242$ & $12 / 12$ & 100 \\
\hline
\end{tabular}




\subsubsection{Construct Validity}

As KMO value is 0.857 , Bartlett's spherical test value is $6280.069(\mathrm{df}=190), P<0.001$. Therefore, the data is suitable for factor analysis. According to the eigenvalue value greater than 1 , four principal components are extracted. The factor load of each item is $0.57-0.71$, and the cumulative contribution rate is $51.924 \%$.(the load of each dimension and eigenvalue are shown in Table 5, and the gravel diagram of each dimension and eigenvalue is shown in Figure 1.

\section{Discussion}

This study finds that the psychometric performance of LBSU applied to college students in Guangdong Province is good, which is consistent with the results of previous similar literature ${ }^{[3-5,9-12,15]}$, suggesting that LBSU is suitable for the assessment of learning burnout of college students in Guangdong Province.

First, the results of internal consistency reliability, split half reliability, construct validity (convergent validity, discriminant validity, principal component analysis) and ceiling / floor effect show that LBSU has good psychometric performance. The internal consistency reliability of the total scale and each dimension of LBSU are above .73; the split half reliability of the total scale was 0.90 , and the split half reliability of each dimension is $0.79-0.87$, which is consistent with the results of previous studies ${ }^{[3-5,15,19-21]}$. It is suggested that the split half reliability and internal consistency reliability of LBSU applied to college students in Guangdong Province are good.

Second, we find that each item of LBSU is highly correlated with its dimension, and each dimension is also

Table 5. principal component analysis and dimensional load of 20 items $(>0.5)$

\begin{tabular}{|c|c|c|c|c|c|c|c|}
\hline \multicolumn{2}{|c|}{ 1st principal compnnent } & \multicolumn{2}{|c|}{ 2nd principal compnnent } & \multicolumn{2}{|c|}{$\underline{\text { 3rd principal compnnent }}$} & \multicolumn{2}{|c|}{ 4th principal compnnent } \\
\hline item & dimension load & item & dimension load & item & $\overline{\text { dimension load }}$ & item & $\overline{\text { dimension load }}$ \\
\hline 5 & .549 & 8 & .535 & 6. & 559 & 13 & .504 \\
\hline 7 & .693 & 10 & .644 & 11 & .565 & 15 & .548 \\
\hline 9 & .585 & 14 & .516 & & & 18 & .573 \\
\hline 12 & .592 & 16 & .590 & & & & \\
\hline 17 & .569 & 19 & .649 & & & & \\
\hline 20 & .522 & & & & & & \\
\hline
\end{tabular}

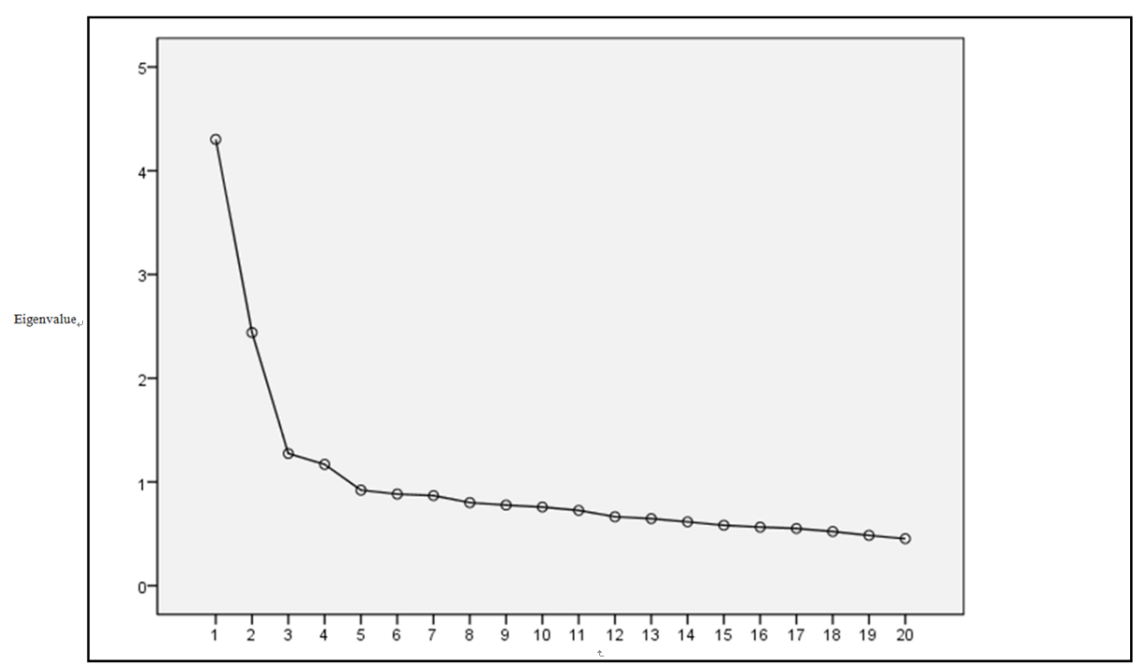

number of components.

Figure 1. Results of principal component analysis for 20 items in LBSU 
highly correlated with the total scale, which is consistent with the results of previous study ${ }^{[15]}$. Principal component analysis is used to analyze the scores of 20 items of LBSU, and four principal components are extracted, with a cumulative contribution rate of $51.924 \%$, indicating that LBSCU has good construct validity. However, the structure of the four principal components extracted in this study is not consistent with the theoretical conception of the three dimensions of the original scale. The reason is that the $2^{\text {nd }}$ item in the first dimension of the original scale, "I think what I am learning is useless", and the three items in the third dimension, "I am very interested in my major", "it is easy for me to get a bachelor's degree" and "I am full of energy when I study" are classified into a new dimension (the fourth dimension) in this study. Further examination of the above four items shows that they can better reflect the connotation of "learning adaptability", rather than just "low mood" or "low sense of achievement".

Third, this study finds that the correlation coefficient between each item and its dimension is $\geq 0.4$, and the correlation coefficients between each item and other dimensions are less than the correlation coefficients between the same item and its dimension. Both the calibration success rate of convergent validity and discriminant validity are $100 \%$, indicating that LBSU has good convergent validity and discriminant validity.

Final, the total score of LBSU and the score of each dimension of this group of college students are normal distribution, without any ceiling effect or floor effect, which is consistent with the results of previous literature ${ }^{[15,18,20]}$, indicating that the items of LBSU are properly selected (typical behavior sampling), and the scale is reasonable and responsive, so the scores tend to be normal distribution.

\section{References}

[1] Seo, J.-H., Kim, B-J., Lee, S.-J., et al. Educational and relational stressors associated with burnout in Korean medical students [J]. Psychiatry Investigation, 2015, 12:451-458.

[2] Cecil, J., McHale, C., Hart, J., et al. Behaviour and burnout in medical students [J]. Medical Education Online, 2014,19: 306-342.

[3] Li Jun, Yin Liqin, Liu Xiaoming. The influence of core self-evaluation on learning burnout of sports majors: the mediating role of coping style [J]. Sports science and technology, 2014, 36 (3): 94-96.

[4] Lu Sixin, Li Lixia, Ke Binbin, et al. Logistic regression analysis on learning burnout and its influencing factors of college students in Guangzhou[J]. School health in China,2014, 35(1):120-122.

[5] Hou Yongmei, Hu Peicheng, Wang Yiyang. The influence of learning burnout on medical students' learning burnout: the mediating role of coping style $[\mathrm{J}]$ Progress in psychology, 2017, 7(7): 845-851.

[6] Yu Xinyue, Yin Mengyan, Zhao Yafei, et al. A cross-sectional historical study on the changes of learning burnout of Chinese college students [J]. Psychological Technology and Application, 2020, 8(2): 74-83.

[7] Dyrbe, L.N., Massie, F.S., Eacker, A., et al. Relationship between burnout and professional conduct and attitudes among US Medical students [J]. JAMA, 2010, 304: 1173-1180.

[8] Dyrbe, L.N., Harper, W., Moutier, C., et al. A multi-instivational study exploring the impact of positive mental health on medical students' professionalism in an era of high burnout [J]. Academic Medicine, 2012, 87:1024-1031.

[9] Zeng Xiaoying. Research exploring the relationship between academic self-efficacy, self-esteem and learning burnout of college students [J]. Journal of Chengdu Normal University, 2014, 30 (12): 90-94.

[10] Qian Kangjie, Yin Keli, Zhang Lirong. The predictive effect of learning burnout on the positive and negative psychological status of college students $[\mathrm{J}]$. Chinese Journal of Mental Health, 2015, 29 (3): 236238.

[11] He Xiangyi. Relationship between learning burnout and academic performance of students in local normal universities [J]. Modern Education Management, 2011, 37(1): 72-74.

[12] Zhou Jianming. Research on the relationship between knowledge learning burnout and entrepreneurial alertness and entrepreneurial performance [J]. Journal of Hubei University of economics, 2014, 11(8): 174-178.

[13] Ye Zhanhang. New research progress on structural dimensions of college students' learning burnout [J]. Social Psychological Science, 2012, 18(4): 59-65.

[14] Zhao Li. Research progress on learning burnout of college students at home and abroad in recent ten years [J]. Literature and Education Materials, 2017, 14(4): 104-106.

[15] Lian Rong, Yang Lixian, Wu Lanhua. The relationship between College Students' professional commitment and learning burnout and the development of the scale [J]. Acta Psychologica Sinica, 2005, 37(5): 632-636.

[16] Cohen J. Statistical power analysis [J]. Curr Direct Psychol Sci, 1992, 1(3): 98-101. 
[17] Zhang Houcan, Xu Jianping. Modern psychology and educational statistics $[\mathrm{M}]$. Beijing: Beijing Normal University Publishing Group, 2012.

[18] Wang Yi, Xiao Huiwen, Zhang Xiaotian, et al. The role of active coping in the relationship between learning burnout and sleep quality among college students in China [J]. Frontiers in Psychology, 2020,11: 647.

[19] Wu Yueping, Yang Qiuxia, Lu Xiaoke, et al. The mediating role of academic procrastination between time management disposition and learning burnout of medical students in a university in Henan Province [J]. Medicine and Society, 2018, 31(9): 66-68.

[20] Li Kang, Tang Li. Survey on learning burnout of college students [J]. Health Vocational Education, 2018, 36(15): 121-122.

[21] Wang Jiankun, Chen Jian, Hao Xiujuan, et al. The influence of learning burnout on life satisfaction of college students -- The mediating role of perceived social support and psychological capital [J]. Chinese Journal of Mental Health, 2018, 32(6): 526-530. 